P.J.Antsaklis, K.M.Passino and S.J.Wang, "An Introduction to Autonomous Control Systems," P roc of $\mathrm{t}$ he 5 th I EEE I nternational S ymposium o $\mathrm{n}$ I ntelligent C ontrol , pp.21-26, Philadelphia, Pennsylvania, Sept. $5-7,1990$

\title{
AN INTRODUCTION TO AUTONOMOUS CONTROL SYSTEMS
}

\author{
P.J. Antsaklis, K.M. Passino \\ Dept. of Electrical Engineering \\ University of Notre Dame \\ Notre Dame, IN 46556
}

S.J. Wang

Jet Propulsion Laboratory

California Inst. of Technology

Pasadena, CA 91109
ABSTRACT

An introduction to the area of intelligent autonomous hierarchical control is given. Autonomous control systems are designed to perform well under significant uncertainties in the system and environment for extended periods of time, and they must be able to compensate for system failures without external intervention. Intelligent autonomous control systems use techniques from the field of Artificial Intelligence to achieve this autonomy. Such control systems evolve from conventional control systems by adding intelligent components, and their development requires interdisciplinary research. The fundamental issues in autonomous control system modelling and analysis are discussed, with emphasis on mathematical modelling. Some recent results in relevant research areas are summarized.

\subsection{INTRODUCTION}

Autonomous means having the power for self government. Autonomous controllers have the power and ability for self governance in the performance of control functions. They are composed of a collection of hardware and software, which can perform the necessary control functions, without external intervention, over extended time periods. To achieve autonomy, the controller must be able to perform a number of functions in addition to the conventional control functions such as tracking and regulation. These additional functions, which include the ability to tolerate failures, are discussed in this paper.

There are several degrees of autonomy. A fully autonomous controller should perhaps have the ability to even perform hardware repair, if one of its components fails. Note that conventional fixed controllers can be considered to have a low degree of autonomy since they can only tolerate a restricted class of plant parameter variations and disturbances. The autonomous controller functional architecture for future space vehicles described in this paper provides the functions to attain a high level of autonomy. It can interface with both the crew, ground station and the on-board systems of the space vchicle. A command by the pilot or the ground station is executed by dividing it into appropriate subtasks which are then performed by the controller. The controller can deal with unexpected situations, new control tasks, and failures within limits. To achieve this, high level decision making techniques for reasoning under uncertainty and taking actions must be utilized. These techniques, if used by humans, are attributed to intelligent behavior. Hence, one way to achieve autonomy is to utilize high level decision making techniques, "intelligent" methods, in the autonomous controller. Autonomy is the objective, and "intelligent" controllers are one way to achieve it. The field of Artificial Intelligence [Charniak and McDermott 85; Shapiro 87] and Operations Research offer some of the tools to add the higher level decision making abilities.

Functions of Autonomous Control Systems: Autonomous control systems must perform well under significant uncertainties in the plant and the environment for extended periods of time and they must be able to compensate for system failures without external intervention. Such autonomous behavior is a very desirable characteristic of advanced systems. An autonomous controller provides high level adaptation to changes in the plant and environment. To achieve autonomy the methods used for control system design should utilize both (i) algorithmic-numeric methods, based on the state of the art conventional control, identification, estimation, and communication theory, and (ii) decision making-symbolic methods, such as the ones developed in computer science and specifically in the field of Artificial Intelligence (AI). In addition to supervising and tuning the control algorithms, the autonomous controller must also provide a high degree of tolerance to failures. To ensure system reliability, failures must first be detected, isolated, and identified, and subsequently a new control law must be designed if it is deemed necessary. The autonomous controller must be capable of planning the necessary sequence of control actions to be taken to accomplish a complicated task. It must be able to interface to other systems as well as with the operator, and it may need learning capabilities to enhance its performance while in operation.

Advanced planning, learning, and expert systems, among others, must work together with conventional control systems in order to achieve autonomy. The need for quantitative methods to model and analyze the dynamical behavior of such autonomous systems presents significant challenges well beyond current capabilities. It is clear that the development of autonomous controllers requires significant interdisciplinary research effort as it integrates concepts and methods from areas such as Control, Identification, Estimation, and Communication Theory, Computer Science, especially Artificial Intelligence, and Operations Research.

Autonomous controllers can of course be used in a variety of systems from manufacturing to unmanned space, atmospheric, ground, and underwater exploratory vehicles. In this paper, we present an autonomous controller architecture for future space vehicles. Referring to a particular class of control problems has the advantage that the development addresses relatively well defined control needs rather than abstract requirements. Furthermore, the autonomous control of space vehicles is highly demanding; consequently the developed architecture is general enough to encompass all related autonomy issues. It should be stressed that all the results presented here apply to any autonomous control system. In other classes of applications, the architecture, or parts of it, can be used directly and the same fundamental concepts and characteristics identified here are valid. In this paper, a shortened version of [Antsaklis, Passino, Wang $88,89]$ the architecture of autonomous controllers necessary for the operation of future advanced space vehicles is presented. The concepts and methods needed to successfully design such an autonomous controller are introduced and discussed. A hierarchical functional autonomous controller architecture is described; it is designed to ensure the autonomous operation of the control system and it allows interaction with the pilot/ground station and the systems on board the autonomous vehicle.

Autonomous controllers are evolutionary and not revolutionary. They evolve from existing controllers in a natural way fueled by actual needs, as it is now discussed:

Design Methodology - History: Conventional control systems are designed using mathematical models of physical systems. A mathematical model which captures the dynamical behavior of interest is chosen and then control design techniques are applied, aided by CAD packages, to design the mathematical model of an appropriate controller. The controller is then realized via hardware or software and it is used to control the physical system. The procedure may take several iterations. The mathematical model of the system must be "simple enough" so that it can be analyzed with available mathematical techniques, and "accurate enough" to describe the important aspects of the relevant dynamical behavior. It approximates the behavior of a plant in the neighborhood of an operating point.

The first mathematical model to describe plant behavior for control purposes is attributed to J.C. Maxwell who in 1868 used differential equations to explain instability problems encountered with James Watt's flyball governor; the governor was introduced in 1769 to regulate the speed of steam engine vehicles. Control theory made significant strides in the past 120 years, with the use of frequency domain methods and Laplace transforms in the 30 's and 40 's and the introduction of the state space analysis in the 60 's. Optimal control in the 50 's and 60 's, stochastic, robust and adaptive control methods in the 60's to today, have made it possible to control more accurately significantly more complex dynamical systems than 
P.J.Antsaklis, K.M.Passino and S.J.Wang, "An Introduction to Autonomous Control Systems," P roc of $\mathrm{t}$ he 5 th I EEE I nternational S ymposium o $\mathrm{n}$ I ntelligent C ontrol , pp.21-26, Philadelphia, Pennsylvania, Sept. $5-7,1990$

the original flyball governor.

The control methods and the underlying mathematical theory were developed to meet the ever increasing control needs of our technology. The evolution in the control area was fueled by three major needs:

(i) The need to deal with increasingly complex dynamical systems.

(ii) The need to accomplish increasingly demanding design requirements.

(iii) The need to attain these design requirements with less precise advanced knowledge of the plant and its environment, that is, the need to control under increased uncertainty.

The need to achieve the demanding control specifications for increasingly complex dynamical systems has been addressed by using more complex mathematical models such as nonlinear and stochastic ones, and by developing more sophisticated design algorithms for, say, optimal control. The use of highly complex mathematical models however, can seriously inhibit our ability to develop control algorithms. Fortunately, simpler plant models, for example linear models, can be used in the control design, this is possible because of the feedback used in control which can tolerate significant model uncertainties. Controllers can then be designed to mee the specifications around an operating point, where the linear model is valid and then via a scheduler a controller emerges which can accomplish the control objectives over the whole operating range. This is, for example, the method typically used for aircraft flight control. In autonomous control we need to significantly increase the operating range of the plant. We must be able to deal with significant uncertainties in models of increasingly complex dynamical systems in addition to increasing the validity range of our control methods. This will involve the use of intelligent decision making processes to generate control actions so that a performance level is maintained even though there are drastic changes in the operating conditions.

There are needs today that cannot be successfully addressed with the existing conventional control theory. They mainly pertain to the arca of uncertainty. Heuristic methods may be needed to tune the parameters of an adaptive control law. New control laws to perform novel control functions should be designed while the system is in operation. Leaming from past experience and planning control actions may be necessary. Failure detection and identification is needed. These functions have been performed in the past by human operators. To increase the speed of response, to relieve the pilot from mundane tasks, to protect operators from hazards, autonomy is desired. It should be pointed out that several functions proposed in late sections, to be part of the autonomous controller, have been performed in the past by separate systems; examples include fault trees in chemical process control for failure diagnosis and hazard analysis, and control system design via expert systems.

In Section 2 the functions, characteristics, and benefits of autonomous control are outlined. Next it is explained that plant complexity and design requirements dictate how sophisticated a controller must be. From this it can be seen that often it is appropriate to use methods from Operations Research or Computer Science to achieve autonomy. Such methods are studied in intelligent control theory. An overview of some relevant research literature in the field of intelligent autonomous control is given togethe with references that outline research directions. An autonomous contro functional architecture for future space vehicles is then presented, which incorporates the concepts and characteristics described earlier. The controller is hierarchical, with three levels, the Execution Level (lowest level), the Coordination Level (middle level), and the Management and Organization Level (highest level). The general characteristics of the overall architecture, including those of the three levels are explained, and an example to illustrate their functions is given.

In Section 3, fundamental issues and attributes of intelligent autonomous systems are described.

Section 4 discusses mathematical models for autonomous systems including "logical" Discrete Event System models. An approach to the quantitative, systematic modelling, analysis, and design of autonomous controllers is also discussed. It is a "hybrid" approach since it is proposed to use both conventional analysis techniques based on difference and differential equations, together with new techniques for the analysis of systems described with a symbolic formalism such as finite automata. The more global, macroscopic view of dynamical systems, taken in the development of autonomous controllers, suggests the use of a model with a hybrid or nonuniform structure, which in turn requires the use of a hybrid analysis.

In Section 5, several major relevant research areas are indicated. In particular, some interesting recent results from the areas of Planning and Expert systems, Machine Learning, Artificial Neural Networks and the area of Restructurable Controls are briefly outlined. Finally, some concluding remarks are given in Section 6.

\section{FUNCTIONAL ARCHITECTURE OF AN AUTONOMOUS CONTROLLER}

\subsection{Intelligent Autonomous Control}

Motivation: Sophistication and Complexity in Control: The complexity of a dynamical system model and the increasingly demanding closed loop system performance requirements, necessitate the use of more complex and sophisticated controllers. For example, highly nonlinear systems normally require the use of more complex controllers than low order linear ones when goals beyond stability are to be met. The increase in uncertainty, which corresponds to the decrease in how well the problem is structured or how well the control problem is formulated, and the necessity to allow human intervention in control, also necessitate the use of increasingly sophisticated controllers. Controller complexity and sophistication is then directly proportional to both the complexities of the plant model and of the control design requirements.

Based on these ideas, [Saridis 79, Gevarter 84 ] suggest a hierarchical ranking of increasing controller sophistication on the path to intelligen controls. At the lowest level, deterministic feedback control based on conventional control theory is utilized for simple linear plants. As plant complexity increases, such controllers will need for instance, state estimators. When process noise is significant, Kalman or other filters may be needed. Also, if it is required to complete a control task in minimum time or with minimum energy, optimal control techniques are utilized. When there are many quantifiable, stochastic characteristics in the plant stochastic control theory is used. If there are significant variations of plant parameters, to the extent that linear robust control theory is inappropriate, adaptive control techniques are employed. For still more complex plants, self-organizing or learning control may be necessary. At the highest level in their hierarchical ranking, plant complexity is so high, and performance specifications so demanding, that intelligent control techniques are used.

In the hierarchical ranking of increasingly sophisticated controllers described above, the decision to choose more sophisticated control techniques is made by studying the control problem using a controller of a certain complexity belonging to a certain class. When it is determined that the class of controllers being studied (e.g., adaptive controllers) is inadequate to meet the required objectives, a more sophisticated class of controllers (e.g. intelligent controllers) is chosen. That is, if it is found that certain higher level decision making processes are needed for the adaptive controller to meet the performance requirements, then these processes can be incorporated via the study of intelligent control theory. These intelligent autonomous controllers are the next level up in sophistication. They are enhanced adaptive controllers, in the sense that they can adapt to more significant global changes in the vehicle and its environment than conventional adaptive controllers, while meeting more stringent performance requirements.

One turns to more sophisticated controllers only if simpler ones cannot meet the required objectives. The need to use intelligent autonomous control stems from the need for an increased level of autonomous decision making abilities in achieving complex control tasks. In the next section a number of intelligent control research results which have appeared in the literature are outlined.

A Literature Overview: In [Antsaklis, Passino and Wang 88,89 ] the authors provided a relatively complete list of references for the field of autonomous control. Here we provide references which we feel will provide the reader with an introduction to autonomous control. First, there are several relevant books: Hierarchical systems are treated in [Mesarovic,Macko and Takahara 70; Findeisen et al.80]. In [Firschein et al.86] the authors explain how a wide variety of AI techniques will be useful in enhancing space station autonomy, capability, safety, etc. Aerospace applications are also discussed in [Heer and Lum 88]. For a book on AI and autonomous systems see [Dougherty and Giardina 88], and for one on cybernetics and intelligent systems see [Glorioso and Osorio 80]. For a book on intelligent manufacturing systems see [Kusiak 90].

Joumals with papers relevant to the area of intelligent autonomous control are The Journal of Intelligent and Robotic Systems, IEEE Trans. on Systems, Man, and Cybemetics, IEEE Transactions on Pattern Analysis and Machine Intelligence, Journal of Intelligent Systems, Journal of Applied Artificial Intelligence, and the standard $f . I$ and control theoretic journals. The reader should also consult some of the recent conference proceedings: Proceedings of the 1985 IEEE Workshop on Intelligent Control, 1986 Intelligent Autonomous Systems Conference, the Space Telerobotics Workshop, and the Proceedings of the IEEE International Symposium on 
P.J.Antsaklis, K.M.Passino and S.J.Wang, "An Introduction to Autonomous Control Systems," P roc of t he 5 th I EEE I nternational S ymposium o $n$ I ntelligent C ontrol , pp.21-26, Philadelphia, Pennsylvania, Sept. 5-7, 1990 .

Intelligent Control in 1987, 1988, 1989, and 1990.

In [Antsaklis, Passino and Wang 88,89] the authors introduce an intelligent autonomous controller and discuss in detail the fundamental characteristics of autonomous control. In [Ozguner 89] the author offers a decentralized control-theoretic view on intelligent control. Fundamentals of intelligent systems such as the principle of increasing intelligence with decreasing precision, are discussed in [Saridis 85], [Saridis 87], and [Meystel 85]. The work in [Saridis $80,83,85,87,89$ ], and [Valavanis 86 ], [Valavanis and Saridis $87 \mathrm{a}, 87 \mathrm{~b}$ ] probably represents the most complete mathematical approach to the analysis of intelligent machines. There have been numerous studies on the use of Expert Systems to control various processes; in [Astrom et al. 86] expert systems have been used in chemical process control. There are interesting relationships between the type of problem examined in intelligent autonomous control, "Fuzzy Control" [Zadeh 88] and "Automated Reasoning" [Wos 88]

\subsection{An Intelligent Autonomous Control Architecture For Future Space}

Vehicles

Here, a functional architecture of an autonomous controller for future space vehicles is introduced and discussed. This hierarchical architecture has three levels, the Execution Level, the Coordination Level, and the Management and Organization Level. The architecture exhibits certain characteristics, as discussed below, which have been shown in the literature to be necessary and desirable in autonomous systems. Based on this architecture we identify the important fundamental issues and concepts that are needed for an autonomous control theory.

Architecture Overview: Structure and Characteristics: The overall functional architecture for an autonomous controller is given by the architectural schematic of Figure 1; for more detailed description see [Antsaklis, Passino and Wang 88,89]. This is a functional architecture rather than a hardware processing one, therefore it does not specify the arrangement and duties of the hardware used to implement the functions described. Note that the processing architecture also depends on the characteristics of the current processing technology; centralized or distributed processing may be chosen for function implementation depending on available computer technology.

The architecture in Figure 1 has three levels. At the lowest level, the Execution Level, there is the interface to the vehicle and its environment via the sensors and actuators. At the highest level, the Management and Organization Level, there is the interface to the pilot and crew, ground station, or onboard systems. The middle level, called the Coordination Level, provides the link between the Execution Level and the Management Level. Note that we follow the somewhat standard viewpoint that there are three major levels in the hierarchy. It must be stressed that the system may have more or fewer than three levels. For instance, see the architecture developed in [Tumer 84]. Some characteristics of the system which dictate the number of levels are the extent to which the operator can intervene in the system's operations, the degree of autonomy or level of intelligence in the various subsystems, the dexterity of the subsystems, the hierarchical characteristics of the plant. Note however that the three levels shown here in Figure 1 are applicable to most architectures of autonomous controllers, by grouping together sublevels of the architecture if necessary. Notice that as it is indicated in the Figure, the lowest, Execution Level involves conventional control algorithms, while the highest, Management and Organization Level involves only higher level, intelligent, decision making methods. The middle, Coordination Level is the level which provides the interface between the actions of the other two levels and it uses a combination of conventional and intelligent decision making methods.

The sensors and actuators are implemented mainly with hardware. They are the connection between the physical system and the controller. Software and perhaps hardware are used to implement the Execution Level. Mainly software is used for both the Coordination and Management Levels. There are multiple copies of the control functions at each level, more at the lower and fewer at the higher levels. For example, there may be one control manager which directs a number of different adaptive control algorithms to control the flexible modes of the vehicle via appropriate sensors and actuators. Another control manager is responsible for the control functions of a robot arm for satellite repair. The control executive issues commands to the managers and coordinates their actions.

Note that the autonomous controller is only one of the autonomous systems on the vehicle. It is responsible for all the functions related to the control of the physical system and allows for continuous online development of the autonomous controller and to provide for various phases of mission operations. The tier structure of the architecture allows us to build on existing advanced control theory. Development progresses, creating each time, higher level adaptation and a new system which can be operated and tested independently. The autonomous controller performs many of the functions currently performed by the pilot, crew, or ground station. The pilot and crew are thus relieved from mundane tasks and some of the ground station functions are brought aboard the vehicle. In this way the vehicle becomes more autonomous.

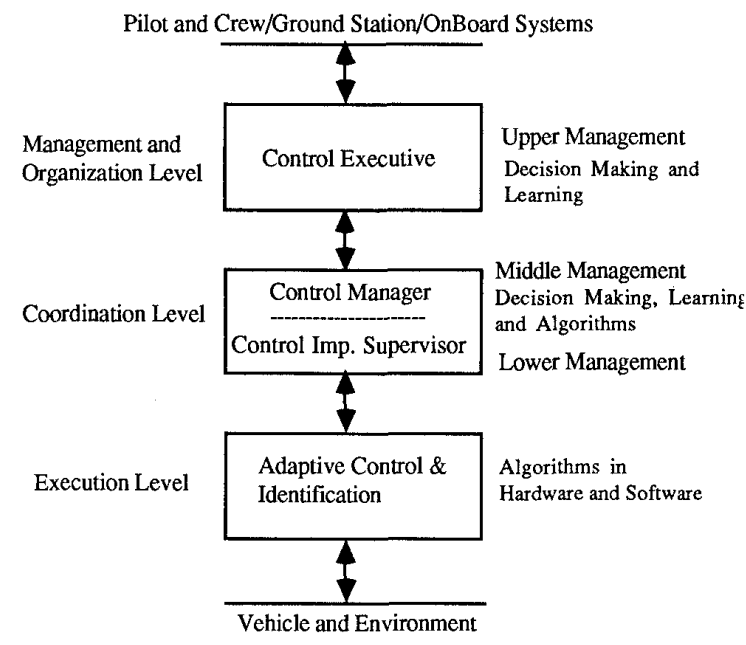

Figure 1. Autonomous Controller Functional Architecture

Functional Operation: Commands are issued by higher levels to lower levels and response data flows from lower levels upwards. Parameters of subsystems can be altered by systems one level above them in the hierarchy. There is a delegation and distribution of tasks from higher to lower levels and a layered distribution of decision making authority. At each level, some preprocessing occurs before information is sent to higher levels. If requested, data can be passed from the lowest subsystem to the highest, e.g. for display. All subsystems provide status and health information to higher levels. Human intervention is allowed even at the control implementation supervisor level, with the commands however passed down from the upper levels of the hierarchy.

The specific functions at each level are described in detail in [Antsaklis, Passino and Wang88,89]. Here we present a simple illustrative example to clarify the overall operation of the autonomous controller. Suppose that the pilot desires to repair a satellite. After dialogue with the control executive, the task is refined to "repair satellite using robot $A$ ". This is arrived at using the capability assessing, performance monitoring, and planning functions of the control executive. The control executive decides if the repair is possible, under the current performance level of the system, and in view of near term planned functions. The control executive, using its planning capabilities, sends a sequence of subtasks sufficient to achieve the repair to the control manager. This sequence could be to order robot A to: "go to satellite at coordinates xyz", "open repair hatch", "repair". The control manager, using its planner, divides say the first subtask, "go to satellite at coordinates xyz", into smaller subtasks: "go from start to $\mathrm{x}_{1} \mathrm{y}_{1} \mathrm{z}_{1}$ ", then "maneuver around obstacle", "move to $\mathrm{x}_{2} \mathrm{y}_{2} \mathrm{z}_{2}$ ",.., "arrive at the repair site and wait". The other subtasks are divided in a similar manner. This information is passed to the control implementation supervisor, which recognizes the task, and uses stored control laws to accomplish the objective. The subtask "go from start to $\mathrm{x}_{1} \mathrm{y}_{1} \mathrm{z}_{1}$ ", can for example, be implemented using stored control algorithms to first, proceed forward 10 meters, to the right 15 degrees, etc. These control algorithms are executed in the controller at the Execution Level utilizing sensor information; the control actions are implemented via the actuators.

\subsection{Some Design Guidelines for A utonomous Controllers}

There are certain functions, characteristics, and behaviors that autonomous systems should possess [Turner et al.84, Firschein et al.86]. These are outlined below. Some of the important characteristics of autonomous controllers are that they relieve humans from time consuming mundane tasks thus increasing efficiency, enhance reliability since they monitor health of the system, enhance performance, protect the system from 
P.J.Antsaklis, K.M.Passino and S.J.Wang, "An Introduction to Autonomous Control Systems," P roc of t he 5 th I EEE I nternational S ymposium o $n$ I ntelligent C ontrol , pp.21-26, Philadelphia, Pennsylvania, Sept. $5-7,1990$

internally induced faults, and they have consistent performance in accomplishing complex tasks.

There are autonomy guidelines and goals that should be followed and sought after in the development of an autonomous system. Autonomy should reduce the work load requirements of the operator or, in the space vehicle case discussed here, of the pilot/crew/ground station, for the performance of routine functions, since the gains due to autonomy would be superficial if the maintenance and operation of the autonomous controller taxed the operators. Autonomy should enhance the functional capability of the system. Since the autonomous controller will be performing the simpler routine tasks, persons will be able to dedicate themselves to even more complex tasks.

There are certain autonomous system architectural characteristics that should be sought after in the design process. The autonomous control architecture should be amenable to evolving future needs and updates in the state of the art. The autonomous control architecture should be functionally hierarchical; for lower level subsystems to take some actions, they have to clear it with a higher level authority. The system must, however, be able to have lower level subsystems, that are monitoring and reconfiguring for failures, act autonomously to certain extent to enhance system safety.

There are also certain operational characteristics of autonomous controllers. Persons should have ultimate supervisory override control of autonomy functions. Autonomous activities should be highly visible, "transparent", to the operator the maximum extent possible.

Finally, there must be certain features inherent in the autonomous system design. Autonomous design features should prevent failures that would jeopardize the overall system mission goals or safety. These features should enhance safety, and avoid false alarms and unnecessary hardware reconfiguration. This implies that the controller should have self-test capability. Autonomous design features should also be tolerant of transient errors, they should not degrade the reliability or operational lifetime of functional elements, they should include adjustable fault detection thresholds, avoid irreversible state changes, and provide protection from erroneous or invalid external commands.

\subsection{CHARACTERISTICS OF AUTONOMOUS CONTROL SYSTEMS}

Based on the architecture described in Section 2 we identify the important fundamental concepts and characteristics that are needed for an autonomous control theory. Note that several of these have been discussed in the literature as outlined above. Here, these characteristics are brought together for completeness. Furthermore, the fundamental issues which must be addressed for a quantitative theory of intelligent autonomous control are introduced and discussed.

There is a successive delegation of duties from the higher to lower levels; consequently the number of distinct tasks increases as we go down the hierarchy. Higher levels are concerned with slower aspects of the system's behavior and with its larger portions, or broader aspects. There is then a smaller contextual horizon at lower levels, i.e, the control decisions are made by considering less information Also notice that higher levels are concerned with longer time horizons than lower levels. Due to the fact that there is the need for high level decision making abilities at the higher levels in the hierarchy, there is increasing intelligence as one moves from the lower to the higher levels. This is reflected in the use of fewer conventional numeric-algorithmic methods at higher levels as well as the use of more symbolic-decision making methods. This is the "principle of increasing intelligence with decreasing precision" by [Saridis 89 ]. The decreasing precision is reflected by a decrease in time scale density, decrease in bandwidth or system rate, and a decrease in the decision (control action) rate. All these characteristics lead to a decrease in granularity of models used, or equivalently, to an increase in model abstractness. Model granularity also depends on the dexterity of the autonomous controller as discussed in [Antsaklis, Passino and Wang 88,89]. The Execution Level of a highly dexterous controller is very sophisticated and it can accomplish complex control tasks. The control implementation supervisor can issue high level commands to a dexterous controller, or it can completely dictate each command in a less dexterous one. The simplicity, and level of abstractness of macro commands in an autonomous controller depends on its dexterity. The more sophisticated the Execution Level is, the simpler are the commands that the control implementation supervisor needs to issue. Notice that a very dexterous robot arm may itself have a number of autonomous functions. If two such dexterous arms were used to complete a task which required the coordination of their actions then the arms would be considered to be two dexterous actuators and a new supervisory autonomous controller would be placed on top for the supervision and coordination task.
In general, this can happen recursively, adding more intelligent autonomous controllers as the lower level tasks, accomplished by autonomous systems, need to be supervised.

There is an ongoing evolution of the intelligent functions of an autonomous controller and this is now discussed. It was pointed out in Section 2 that complex control problems required a controller sophistication that involved the use of AI methodologies. It is interesting to observe the following [Mendel and Zapalac 68]: Although there are characteristics which separate intelligent from non-intelligent systems, as intelligent systems evolve, the distinction becomes less clear. Systems which were originally considered intelligent evolve to gain more character of what are considered to be non-intelligent, numeric-algorithmic systems. An example is a route planner. Although there are AI route planning systems, as problems like route planning become better understood, more conventional numericalgorithmic solutions are developed. The AI methods which are used in intelligent systems, help us to understand complex problems so we can organize and synthesize new approaches to problem solving, in addition to being problem solving techniques themselves. AI techniques can be viewed as research vehicles for solving very complex problems. As the problem solution develops, purely algorithmic approaches, which have desirable implementation characteristics, substitute AI techniques and play a greater role in the solution of the problem. It is for this reason that we concentrate on achieving autonomy and not on whether the underlying system can be considered "intelligent".

\subsection{MATHEMATICAL MODELS FOR AUTONOMOUS SYSTEMS}

For autonomous control problems normally, the plant is so complex that it is either inappropriate or impossible to describe it with conventiona system models such as differential or difference equations. For instance even though it might be possible to accurately describe some system with very complex nonlinear differential equations, it may be inappropriate if this description makes subsequent analysis too difficult. The complexity of the plant model necessary for design depends on both the complexity of the physical system and on how demanding the design specifications are. There is a tradeoff between model complexity and our ability to perform analysis on the system via the model. However, if the control performance specifications are not too demanding, a more abstract, higher level, model can be utilized, which will make subsequent analysis simpler. This mode intentionally ignores some of the system characteristics, specifically those that need not be considered in attempting to meet the particular performance specifications. For example, a simple temperature controller could ignore almost all dynamics of the house or the office and consider only a temperature threshold model of the system to switch the fumace off or on.

Logical Discrete Event System (DES) models such as those used in the Ramadge-Wonham framework (e.g. [Zhong and Wonham 90]) or such as Petri nets [Peterson 81] are quite useful for modelling the higher leve decision making processes in the intelligent autonomous controller. It was shown in [Passino and Antsaklis 89a,89b] that DES-theoretic models can be used to represent AI planning systems which are an important component of the intelligent autonomous controller. Also, it was shown in [McDonnellDouglas 88] that Petri nets can be used as knowledge representation tools in AI. In particular the authors showed that knowledge that can be represented with semantic networks, scripts, and production rules in an expert system can also be clearly represented with Petri net models. The "timed" or "performance" models from DES-theoretic research will also prove useful in modelling components of the higher levels in the intelligen autonomous controller. For instance, queueing network models, Markov chains, etc. The choice of whether to use such models will, of course depend on what properties of the autonomous system need to be studied.

The quantitative, systematic techniques for modelling, analysis, and design of control systems are of central and utmost practical importance in conventional control theory. Similar techniques for intelligent autonomous controllers do not exist. This is of course because of their novelty, but for the most part, it is due to the "hybrid" structure (nonuniform, nonhomogeneous nature) of the dynamical systems under consideration. The systems are hybrid since in order to examine autonomy issues, a more global, macroscopic view of a dynamical system must be taken than in conventional control theory. Modelling techniques for intelligent autonomous systems must be able to support this macroscopic view of the dynamical system, hence it is necessary to represent both numeric and symbolic information. We need modelling methods that can gather all information necessary for analysis and design. For example, we need to model the dynamical system to be controlled (e.g., a space platform), failures that might occur in the system, the conventional adaptive controller, 
P.J.Antsaklis, K.M.Passino and S.J.Wang, "An Introduction to Autonomous Control Systems," P roc of t he 5 th I EEE I nternational S ymposium o $n$ I ntelligent $C$ ontrol , pp.21-26, Philadelphia, Pennsylvania, Sept. $5-7,1990$

and the high level decision making processes at the management and organization level of the intelligent autonomous controller (e.g., an AI planning system performing actions that were once the responsibility of the ground station). The nonuniform components of the intelligent controller all take part in the generation of the low level control inputs to the dynamical system, therefore they all must be considered in a complete analysis. For an extended discussion on the modelling of hybrid systems consult [Zeigler 87].

It is our belief that research should begin by using different models for different components of the intelligent autonomous controller. Full hybrid models that can represent large portions or even the whole autonomous system should be examined but much can be attained by using the best available models for the various components of the architecture and joining them via some appropriate interconnecting structure. For instance, research in the area of systems that are modelled with a logical DES model at the higher levels and a difference equation at the lower level should be examined. In any case, our modelling philosophy requires the examination of hierarchical models. Much work needs to be done on hierarchical DES modelling, analysis, and design, let alone the full study of hybrid hierarchical dynamical systems. Some research has begun to address hierarchical DES [Zhong and Wonham90].

A practical but very important issue is the simulation of hybrid systems. This requires simulation of both conventional differential equations and symbolic decision making processes or DES. Normally, numeric-algorithmic processing is done with languages like FORTRAN and symbolic decision making can be implemented with LISP or PROLOG while DES are often simulated with SLAM. Sometimes several types of processing are done on computers with quite different architectures. There is then the problem of combining symbolic and numeric processing on one computer. If the computing is done on separate computers, the communication link normally presents a serious bottleneck. Combining $\mathrm{AI}, \mathrm{DES}$, and conventional numeric processing is currently being addressed by many researchers and some promising results have been reported.

\subsection{PLANNING AND EXPERT SYSTEMS, LEARNING AND}

NEURAL NETWORKS, RESTRUCTURABLE CONTROL

In this Section we will discuss results obtained on the analysis and design of several components of the intelligent autonomous controller architecture. One can roughly categorize research in the area of intelligent autonomous control into two areas: conventional control theoretic research, addressing the control functions at the Execution and Coordination Levels, and the modelling, analysis, and design of higher level decision making systems found in the Management and Organization Level, and the Coordination Level. Below we provide only a sampling of the results to introduce the reader to these research areas.

To determine how to utilize AI techniques it is productive to study the relationships between $\mathrm{AI}$ and conventional control methods. In this way one can determine what AI techniques have to offer over conventional control methods. For instance, the authors in [Passino and Antsaklis 89a] have provided a systems and control theoretic perspective on AI planning (and expert) systems. In this work, the authors explain how AI planning systems are in fact control systems where the input and output variables are symbols rather than numbers. It is shown that the techniques used in the implementation of AI planning systems are actually generalized open and closed loop control, state estimation, system identification, and adaptive control.

It is also important to study how to use conventional control techniques in conjunction with AI techniques to perform autonomous control functions. For instance, in [Passino and Antsaklis 88a; Passino 86] the authors introduce a fault detection and identification (FDI) system that is composed of AI decision making mechanisms and conventional FDI algorithms. The "hybrid" algorithmic-decision making FDI system detects and identifies failures for an intelligent restructurable controller on board an advanced aircraft.

Some control theoretic techniques offer modelling, analysis, and design techniques for the higher level decision making mechanisms in the intelligent autonomous controller. For instance, in [Passino and Antsaklis $89 \mathrm{~b}, 88 \mathrm{~b}, \mathrm{c}]$ the authors show that AI planning problems can be studied in a discrete event system (DES) theoretic framework by utilizing the $A^{*}$ algorithm. Moreover, there are many recent results developed in a DEStheoretic framework that can be used for the study of components of the intelligent autonomous controller (e.g., results from the Ramadge-Wonham formulation for the study of "logical" DES models)

It is important to note that in order to obtain a high degree of autonomy it is absolutely necessary to, in some way, adapt or learn [Antsaklis 88] Although the literature on higher level learning performed in conjunction with low level adaptation is limited, in [Gao, Peek and Antsaklis 88; Peek and Antsaklis 89,90] the authors show how an expert learnirg system can be used to tune the parameters of an adaptive controller for a large flexible space antenna. Neural networks also appear to offer methodologies to perform learning functions in the intelligent autonomous controller (see for instance, the April issues of the IEEE Control Systems Magazine in 1987 1988 and the Special Issue of April 1989 [Antsaklis 90] ; also the new IEEE Trans. on Neural Networks). Neural networks can also be used to implement certain components of the intelligent autonomous controller. For instance, the authors in [Sartori, Passino and Antsaklis 89] investigate how to implement the match phase of expert systems with a "multi-layer perceptron".

We stress that in autonomous control we seek only to significantly widen the operating range of the system so that significant failures and environmental changes can occur and performance will still be maintained. All of the conventional control techniques are useful in the development of autonomous controllers and they are relevant to the study of autonomous control. It is the case however, that certain techniques are more suitable for interfacing to the autonomous controller and for compensating for significant system failures. For instance the area of "restructurable" or "reconfigurable" control systems [Passino 86; Stengel 84]. Recently there have been advances in the theory of restructurable controls [Gao and Antsaklis 89,90 ] where the authors develop stability bounds on the allowable parameter variations, induced by system failures.

It is our viewpoint that conventional modelling, analysis, and design methods should be used whenever they are applicable for the components of the intelligent autonomous controller. For instance, they should be used a the Execution Level of many autonomous controllers. We propose to augment and enhance existing theories rather than develop a completely new theory for the hybrid systems described above; we wish to build upon existing, well understood and proven conventional methods. The symbolic/numeric interface is a very important issue; consequently it should be included in any analysis. There is a need for systematically generating less detailed, more abstract models from differential/difference equation models to be used in higher levels of the autonomous controller (Coordination Level). There is also a need for systematically extracting the necessary information from lower level symbolic models to generate higher level symbolic models to be used in the hierarchy where appropriate. Tools for the analysis of this information extraction also need to be developed (see for instance [Passino, Sartori, and Antsaklis 89]). In this way conventiona analysis can be used in conjunction with the developed analysis methods to obtain an overall quantitative, systematic analysis paradigm for intelligent autonomous control systems. In short, we propose to use hybrid modelling, analysis, and design techniques for nonuniform systems. This approach is not unlike the approaches used in the study of any complex phenomena by the scientific and engineering communities.

\subsection{CONCLUDING REMARKS}

The fundamental issues in autonomous control system modelling and analysis were identified and briefly discussed, thus providing an introduction to the research problems in the area. A hierarchical functional autonomous controller architecture was also presented. It was proposed to utilize a hybrid approach to modelling and analysis of autonomous systems. This will incorporate conventional control methods based on differential equations and new techniques for the analysis of systems described with a symbolic formalism. In this way, the well developed theory of conventional control can be fully utilized. It should be stressed that autonomy is the design requirement and intelligent control methods appear, at present, to offer some of the necessary tools to achieve autonomy. A conventional approach may evolve and replace some or all of the "intelligent" functions. Note that this paper is based on the results presented in [Antsaklis, Passino and Wang $88,89]$.

Acknowledgement: This work was partially supported by the Jet Propulsion Laboratory, Pasadena, California.

\subsection{REFERENCES}

[Albus 81] Albus J.,et al, "Theory and Practice of Intelligent Control", Proc. 23rd IEEE COMPCON, pp 19-39, 1981.

[Antsaklis 88] Antsaklis P.J., "Learning in Control", in Panel Discussion on "Machine Learning in a Dynamic World", M.M.Kokar Organizer, Proc. of the 3rd IEEE Intern. Symposium on Intelligent Control, pp. 500-507, Arlington, VA, Aug. 24-26, 1988. 
P.J.Antsaklis, K.M.Passino and S.J.Wang, "An Introduction to Autonomous Control Systems," P roc of t he 5 th I EEE I nternational S ymposium o $n$ I ntelligent C ontrol , pp.21-26, Philadelphia, Pennsylvania, Sept. $5-7,1990$

[Antsaklis 90] Antsaklis P.J., "Neural Networks in Control Systems", Guest Editor's Introduction, IEEE Control Systems Magazine, Vol.10, No.3, pp.3-5, April 1990; Special Issue on 'Neural Networks in Control Systems' of the IEEE Control Systems Magazine, Vol.10, No.3, pp.3-87, April 1990.

[Antsak]is, Passino and Wang 88] Antsaklis P.J., Passino K.M. and Wang S J., "Autonomous Control Systems: Architecture and Fundamental Issues", Proc. of the 1988 American Control Conference, pp. 602-607, Atlanta, Georgia, June 15-17, 1988.

[Antsaklis, Passino and Wang 89] Antsaklis P.J., Passino K.M. and Wang S J., "Towards Intelligent Autonomous Control Systems: Architecture and Fundamental Issues", Journal of Intelligent and Robotic Systems, Vol.1, pp.315-342, 1989 .

[Astrom et al.86] Astrom K.J., et al, "Expert Control", Automatica, Vol. 22, No. 3, pp. $277-286,1986$

Charniak and McDermott 85] Chamiak E. and McDermolt D.,Introduction to Artificial Intelligence,Addison Wesley, Reading Mass, 1985.

[Dougherty and Giardina 88] Dougherty E.R. and Giardina C.R., Mathematical Methods for Artificial Intelligence and Autonomous Systems, Prentice Hall, 1988.

[Findeisen et al.80] Findeisen W.,et al, Control and Coordination in Hierarchical Systems, Wiley, New York, 1980

[Firschein et al.86] Firschein O.,et al, Artificial Intelligence for Space Station Automation, Noyes, New Jersey, 1986.

[Gao and Antsaklis 89] Gao Z. and Antsaklis P. J., "On the Stability of the Pseudo-Inverse Method for Reconfigurable Control Systems", Proc. of the National Aerospace and Electronics Conference, pp. 333-337, Dayton, OH, May 22-26, 1989. Also in the Intern. Journal of Control. To appear.

[Gao and Antsaklis 90] Gao Z. and Antsaklis P. J., "Pseudo-Inverse Methods for Reconfigurable Control with Guaranteed Stability", Proc. of the 1990 IFAC 11th World Congress, Tallinn, USSR, Aug. 13-17, 1990.

[Gao, Peek and Antsaklis 90] Gao Z., Peek M.D. and Antsaklis P.J. "Learning for the Adaptive Control of a Large Flexible Structure", Proc. of the 3rd IEEE International Symposium on Intelligent Control, pp. 508-512, Arlington, VA, Aug. 24-26, 1988.

[Gevarter 84] Gevarter W.B.,Artificial Intelligence, Noyes, NJ, 1984.

[Glorioso and Osorio 80] Glorioso R.M. and Colon Osorio F.C., Engineering Intelligent Systems, Digital Press, 1980.

[Heer and Lum 88] Heer E., Lum H., ed., Machine Intelligence and Autonomy for Aerospace Systems, AIAA, Wash. DC, 1988.

[JPL 87] Antsaklis P.J., Passino K.M., "Autonomous Control Systems: Architecture and Concepts for Future Space Vehicles", Final Report, Jet Propulsion Laboratory Contract No. 957856, October 1987.

[JPL 88] Antsaklis P.J., Gao Z., Passino K.M., Peek M.D., and Sartori, M. "Learning and Decision Making Models for Higher Level Adaptation", Final Report, Jet Propulsion Laboratory Contract No. 957856 Mod. 2, November 1988

[JPL 89] Antsaklis P.J., Peek M.D., Gao Z., Passino K.M., "Learning Contro for Higher Level Adaptation", Final Report, Jet Propulsion Laboratory Contract No. 957856, Mod. 3, November 1989.

[Kusiak 90] Kusiak A., Intelligent Manufacturing Systems, Prentice Hall, 1990.

[McDonnellDouglas 86] Passino K.M.and Antsaklis P.J., "Restructurable Controls Study: An Artificial Intelligence Approach to the Fault Detection and Identification Problem", Final Report for McDonnell Douglas Contract No. Z60110, October 1986 .

[McDonnellDouglas 87] Antsaklis P.J.and Passino K.M., "Artificial Intelligence Planning and Control Theory Relationships", Final Report for McDonnell Douglas Contract No. Z71145, October 1987.

[McDonnellDouglas 88] Antsaklis P.J, Passino K.M. and Sartori M.A. "Modelling and Analysis of Artificial Intelligence Planning Systems", Final Report for McDonnell Douglas Contract No. Z81014, October 1988.

[Mendel and Zapalac 68] Mendel J. and Zapalac J.,"The Application of Techniques of Artificial Intelligence to Control System Design", in Advance in Control Systems, C.T. Leondes, ed., Academic Press, NY, 1968.

[Mesarovic,Macko and Takahara 70] Mesarovic M., Macko D. and Takahara Y., Theory of Hierarchical. Multilevel. Systems, Academic Press, 1970.

[Meystel 85] Meystel A.,"Intelligent Control: Issues and Perspectives", Proc. IEEE Workshop on Intelligent Control, pp 1-15, 1985.

[Ozguner 89] Ozguner U.,"Decentralized and Distributed Control Approaches and Algorithms", Proc. of the 28th IEEE Conference on Decision and Control, pp.1289-1294, Tampa, Florida, Dec 1989.

[Passino 86] Passino K.M., "Restructurable Controls and Artificial Intelligence", McDonnell Aircraft Internal Report, IR-0392, April 1986.

[Passino 89] Passino K.M., Analysis and Synthesis of Discrele Even Regulator Systems, Ph.D. Dissertation, Dept. of Electrical and Computer Eng., Univ. of Notre Dame, April 1989.

[Passino and Antsaklis 88a] Passino K. M. and Antsaklis P. J., "Fault Detection and Identification in an Intelligent Restructurable Controller", Journal of Intelligent and Robotic Systems, Vol. 1, pp.145-161, June 1988.
[Passino and Antsaklis 88b] Passino K. M. and Antsaklis P. J., "Artificial Intelligence Planning Problems in a Petri Net Framework", Proc. of the American Control Conference, pp. 626-631, June 1988.

[Passino and Antsaklis 88c] Passino K. M. and Antsaklis P. J., "Planning via Heuristic Search in a Petri Net Framework", Proc. of the 3rd IEEE International Symposium on Intelligent Control, pp. 350-355, Arlington, VA, Aug. 24-26, 1988.

[Passino and Antsaklis 89a] Passino K. M. and Antsaklis P. J., "A System and Control Theoretic Perspective on Artificial Intelligence Planning Systems", Applied Artificial Intelligence, Vol. 3, pp. 1-32, 1989.

[Passino and Antsaklis 89b] Passino K. M. and Antsaklis P. J., "On the Optimal Control of Discrete Event Systems" , Proc. of the 28th IEEE Conf. on Decision and Control, pp. 2713-2718, Tampa, FL, Dec. 13-15, 1989.

[Passino and Antsaklis 90] Passino K. M. and Antsaklis P. J., "Timing Characteristics of Discrete Event Systems", Control Systems Technical Note \#68, Dept. of Electrical and Computer Engineering, University of Notre Dame, June 1989

[Passino, Sartori and Antsaklis 89] Passino K.M., Sartori M.A. and Antsaklis P.J, "Neural Computing for Numeric to Symbolic Conversion in Control Systems", IEEE Control Systems Magazine, pp. 44-52, April 1989.

[Peek and Antsaklis 89] Peek M.D. and Antsaklis P.J., "Parameter Learning for Performance Adaptation in Large Space Structures", Proc. of the 4th IEEE International Symposium on Intelligent Control, Albany, NY, Sept. 25-27, 1989.

[Peek and Antsaklis 90] Peek M.D. and Antsaklis P.J., "Parameter Leaming for Performance Adaptation", IEEE Control Systems Magazine, December 1990. To appear.

[Peterson 81] Peterson J.L., Petri Net Theory and the Modeling of Systems, Prentice-Hall, Englewood Cliffs, NJ, 1981

[Saridis 79] Saridis G.N.,"Toward the Realization of Intelligen Controls",Proc. of the IEEE, Vol. 67, No. 8, pp. 1115-1133, August 1979.

[Saridis 80] Saridis G.N.,"Intelligent Controls for Advanced Automated Processes",Proc. of the Automated Decision Making and Problem Solving Conf., NASA CP-2180, May 1980.

[Saridis 83] Saridis G.N.,"Intelligent Robot Control", IEEE Trans. on Automatic Control, Vol. AC-28, No. 5, pp. 547-556, May 1983.

[Saridis 85] Saridis G.N.,"Foundations of the Theory of Intelligent Controls", Proc. IEEE Workshop on Intelligent Control, pp 23-28, 1985.

[Saridis 87] Saridis G.N.,"Knowledge Implementation: Structures of Intelligent Control Systems", Proc. IEEE International Symposium on Intelligent Control, pp. 9-17, 1987.

[Saridis 89] Saridis G.N., "Analytic Formulation of the Principle of Increasing Precision with Decreasing Intelligence for Intelligent Machines", Automatica Vol.25, No.3, pp. 461-467, 1989.

[Sartori, Passino and Antsaklis 89] Sartori M. A., Passino K.M. and Antsaklis P.J., "Artificial Neural Networks in the Match Phase of Rule Based Expert Systems", Proc, of the Twenty-seventh Annual Allerton Conference on Communication, Control and Computing, pp 1037-1046, Univ. of Illinois at Urbana-Champaign, Sept. 27-29, 1989.

[Sartori, Passino and Antsaklis 90] Sartori M. A., Passino K.M. and Antsaklis P.J., "An Artificial Neural Network Solution to the Match Phase Problem in Rule Based AI Systems". Submitted for joumal publication.

[Shapiro 87] Shapiro S.C., ed, "Encyclopedia of Artificial Intelligence", Wiley, NY, 1987

[Stengel 84] Stengel R.F., "AI Theory and Reconfigurable Flight Control Systems", Princeton University Report 1664-MAE, June 1984.

[Turner 84] Tumer P.R., et al, "Autonomous Systems: Architecture and Implementation", Jet Propulsion Laboratories, Report No. JPL D-1656, August 1984

[Valavanis 86] Valavanis K.P., A Mathematical Formulation For The Analytical Design Of Intelligent Machines, PhD Dissertation, Electrical and Computer Engineering Dept., Rensselaer Polytechnic Institute, Troy NY, Nov. 1986 .

[Valavanis and Saridis 87a] Valavanis K.P. and Saridis G.N., "Information Theoretic Modelling of Intelligent Robotic Systems, Part I: The Organization Level", Proc. of the 26th Conf. on Decision and Control, Los Angeles, pp. 619-626, Dec. 1987.

[Valavanis and Saridis 87b] Valavanis K.P.,and Saridis G.N., "Information Theoretic Modelling of Intelligent Robotic Systems, Part II: The Coordination and Execution Levels", Proc. of the 26th Conf. on Decision and Control, Los Angeles, pp. 627-633, Dec. 1987.

[Wos 88] Wos L., Automated Reasoning: 33 Basic Research Problems, Prentice Hall, NJ, 1988.

[Zadeh 88] Zadeh L.A., "Fuzzy Logic", Computer, pp. 83-93, April 1988.

[Zeigler 87] Zeigler B.P., "Knowledge Representation from Newton to Minsky and Beyond", Journal of Applied Artificial Intelligence, 1:87-107, 1987.

[Zhong and Wonham 90] Zhong H. and Wonham W.M., "On Consistency of Hierarchical Supervision in Discrete-Event Systems", Systems Control Group Report No.9003, Dept. of Electr. Engr., Univ. of Toronto, Feb. 90. 